IJMMS 25:4 (2001) 239-252

PII. S0161171201004318

http://ijmms.hindawi.com

(C) Hindawi Publishing Corp.

\title{
KREIIN'S TRACE FORMULA AND THE SPECTRAL SHIFT FUNCTION
}

\section{KHRISTO N. BOYADZHIEV}

(Received 15 October 1999 and in revised form 3 January 2000)

\begin{abstract}
Let $A, B$ be two selfadjoint operators whose difference $B-A$ is trace class. Krein proved the existence of a certain function $\xi \in L_{1}(\mathbb{R})$ such that $\operatorname{tr}[f(B)-f(A)]=$ $\int_{\mathbb{R}} f^{\prime}(x) \xi(x) d x$ for a large set of functions $f$. We give here a new proof of this result and discuss the class of admissible functions. Our proof is based on the integral representation of harmonic functions on the upper half plane and also uses the Baker-Campbell-Hausdorff formula.
\end{abstract}

2000 Mathematics Subject Classification. Primary 47A55; Secondary 81Q05, 81U20.

1. Introduction. Kreǐn [19, 20, 21] developed the trace formula

$$
\operatorname{tr}[f(B)-f(A)]=\int_{\mathbb{R}} f^{\prime}(x) \xi(x) d x
$$

which originated from Lifšic [22]. The function $\xi$ is known as Kreǐn's spectral shift function (SSF) and has important applications in spectral theory. For instance, $\xi$ is related to the scattering matrix $S(\lambda)$ for $A$ and $B$ by the remarkable formula [3],

$$
\operatorname{det} S(\lambda)=e^{-2 \pi i \xi(\lambda)}
$$

More recently, Kreĭn's spectral shift function was used for the computation of Witten's index in supersymmetric scattering theory $[7,14]$ and in inverse spectral theory for Schrödinger operators [15]. The trace formula can also be viewed as a mean value theorem for operators [10]. A comprehensive survey and references can be found in [6]. For more recent results see $[4,12,13,18,29]$, and for extensions to non-selfadjoint operators see $[1,16,26]$ and the references therein. Long time Kreĭn's original proof [19] —also in [2, 21, 28, 32] —was the only one available. This proof is based on the relation

$$
\log \operatorname{det}_{B / A}(z)=\int_{\mathbb{R}} \frac{\xi(x)}{x-z} d x
$$

where $\operatorname{det}_{B / A}$ is the perturbation determinant for the pair $A, B$. It uses properties of such determinants and the integral representation of holomorphic functions on the upper half plane with a bounded imaginary part. In 1985, Voiculescu [31] approached the trace formula from a different direction. He constructed explicitly the spectral shift function in the finite dimensional case and then used the quasidiagonality of selfadjoint operators relative to the Hilbert-Schmidt class to extend by approxima- 
tion the trace formula to bounded operators on a separable Hilbert space. Recently, Sinha and Mohapatra [28] applied a sophisticated approximation procedure to extend the formula from bounded to unbounded operators and thus provided an alternative proof of Kreĭn's theorem. Another approach, using contour integration was suggested in [27]. We highly recommend the recent article [4] which contains very interesting comments on several formula representations of the SSF.

OUTLINE OF THE PAPER. We give here a new proof of the trace formula which does not use determinants or approximation. The spectral shift function is defined as the boundary value of one appropriate harmonic function on the upper half plane, see (2.6). This way we provide a new formula representation for the SSF. A special feature of our proof is the connection to the Baker-Campbell-Hausdorff formula in Lemma 1.1.

In Section 2, we state Kreı̌n's theorem. Section 3 contains its proof. In Section 4, we give some examples of admissible functions and in Section 5, we deal with a substitution in the trace formula. The Baker-Campbell-Hausdorff formula is discussed in Section 6 . The paper is accessible to graduate students with a background in functional analysis.

Prerequisite. We work with linear operators on a complex Hilbert space. Throughout $S_{1}$ stands for the trace class. The notation $\|\cdot\|_{1}$ is used for the norm on $S_{1}$ as well as for the norm on $L_{1}(\mathbb{R})$, and $\|\cdot\|$ is the uniform operator norm.

LEMMA 1.1. Let $X, Y$ be two bounded operators with $X+Y \in S_{1}$. If $\|X\|,\|Y\|$ are sufficiently small, then the operator $Z$ defined by $e^{X} e^{Y}=e^{Z}$ belongs to the trace class, and

$$
\operatorname{tr} Z=\operatorname{tr}(X+Y)
$$

The proof is given in Section 6.

We need some simple facts about Poisson integrals and harmonic functions which can be found, for instance, in [17].

LEMMA 1.2. For every $g \in L_{1}(\mathbb{R})$,

$$
g(x)=\lim _{y \rightarrow 0+} \frac{1}{\pi} \int_{\mathbb{R}} g(t) \frac{y}{y^{2}+(x-t)^{2}} d t
$$

almost everywhere. The convergence is everywhere and uniform when $g$ is uniformly continuous.

LEMMA 1.3 (Fatou). If $h(x, y), x \in \mathbb{R}, y>0$ is a bounded harmonic function on the upper half plane, then its nontangential boundary values $h(x)=\lim _{y \rightarrow 0+} h(x, y)$ exist almost everywhere and

$$
h(x, y)=\frac{1}{\pi} \int_{\mathbb{R}} h(t) \frac{y}{y^{2}+(x-t)^{2}} d t .
$$




\section{Kreĭn's trace formula}

DEFINITION 2.1. Let $K$ be the set of all functions with the representation

$$
f(x)=\int_{\mathbb{R}} \frac{e^{i s x}-1}{s} d \mu(s),
$$

where $\mu$ is a finite measure on $\mathbb{R}$.

If $A$ is a selfadjoint operator with spectral resolution $E_{A}(\lambda)$ and $f \in K$, we define as usual

$$
f(A)=\int_{\mathbb{R}} f(\lambda) d E_{A}(\lambda)=\int_{\mathbb{R}} \frac{e^{i s A}-1}{s} d \mu(s) .
$$

LEMmA 2.2. Suppose that $A, B$ are selfadjoint operators and $B-A \in S_{1}$. Then for all $f \in K$ :

$$
f(B)-f(A) \in S_{1} \quad \text { and } \quad\|f(B)-f(A)\|_{1} \leq\|B-A\|_{1}\|\mu\| .
$$

THEOREM 2.3. Let $A$ and $B$ be two selfadjoint operators with $B-A \in S_{1}$. There exists a function $\xi \in L^{1}(\mathbb{R})$ such that

(a) for every $f \in K$,

$$
\operatorname{tr}[f(B)-f(A)]=\int_{\mathbb{R}} f^{\prime}(x) \xi(x) d x .
$$

In particular,

$$
\operatorname{tr}(B-A)=\int_{\mathbb{R}} \xi(x) d x
$$

(b) $\|\xi\|_{1} \leq\|B-A\|_{1}$.

(c) If $A \leq B$, then $0 \leq \xi$ almost everywhere.

(d) $\xi(x)=0$ outside of any interval containing $\sigma(A) \cup \sigma(B)$.

The function $\xi$ is called Kreĭn's spectral shift function (SSF). It can be computed by the formula

$$
\xi(x)=\lim _{y \rightarrow 0+} h(x, y), \quad \text { a.e. } x \in \mathbb{R}
$$

where

$$
h(x, y)=\frac{1}{\pi} \operatorname{tr}\left[\arctan \frac{B-x}{y}-\arctan \frac{A-x}{y}\right]=\frac{1}{2 \pi i} \int_{\mathbb{R}} e^{-i x s-y|s|} \operatorname{tr}\left[\frac{e^{i s B}-e^{i s A}}{s}\right] d s .
$$

We write sometimes $\xi(x)=\xi(x ; A, B)$ to indicate the dependence on $A$ and $B$.

3. Proof of Theorem 2.3. First we prove Lemma 2.2. The relation

$$
e^{i s B}-e^{i s A}=\int_{0}^{s} e^{i(s-t) B}(B-A) e^{i t A} d t
$$

implies

$$
\left\|e^{i s B}-e^{i s A}\right\|_{1} \leq|s|\|B-A\|_{1} .
$$

When $f \in K$, we have

$$
f(B)-f(A)=\int_{\mathbb{R}} \frac{e^{i s B}-e^{i s A}}{s} d \mu(s) .
$$


Therefore $f(B)-f(A) \in S_{1}$ and

$$
\|f(B)-f(A)\|_{1} \leq \int_{\mathbb{R}} \frac{\left\|e^{i s B}-e^{i s A}\right\|_{1}}{|s|} d|\mu|(s) \leq\|B-A\|_{1}\|\mu\| .
$$

We introduce one important tool, the function $g(t)=\arctan t$. It belongs to the class $K$ because the two representations

$$
\arctan t=\int_{0}^{1} \frac{t}{1+t^{2} u^{2}} d u, \quad \frac{t}{1+t^{2} u^{2}}=\frac{t}{2} \int_{\mathbb{R}} e^{i t u s} e^{-|s|} d s
$$

together give

$$
\arctan t=\frac{1}{2 i} \int_{\mathbb{R}} \frac{e^{i s t}-1}{s} e^{-|s|} d s .
$$

For all $x \in \mathbb{R}, y>0$, we define

$$
h(x, y)=\frac{1}{\pi} \operatorname{tr}\left[\arctan \frac{B-x}{y}-\arctan \frac{A-x}{y}\right] .
$$

In view of (3.4) and (3.6),

$$
\pi|h(x, y)| \leq\left\|\arctan \frac{B-x}{y}-\arctan \frac{A-x}{y}\right\|_{1} \leq \frac{1}{y}\|B-A\|_{1} .
$$

Using the representation (3.6) we can write also

$$
h(x, y)=\frac{1}{2 \pi i} \int_{\mathbb{R}} e^{-i x s-y|s|} \operatorname{tr}\left[\frac{e^{i s B}-e^{i s A}}{s}\right] d s
$$

which shows that $h(x, y)$ is harmonic in the upper half plane $x \in \mathbb{R}, y>0$. To find out more about this function we study one special unitary operator.

Set $z=x+i y$ and consider the unitary Cayley transforms:

$$
\begin{aligned}
T_{A} & =(A-\bar{z})(A-z)^{-1}=I+2 i y(A-z)^{-1}, \\
T_{B} & =(B-\bar{z})(B-z)^{-1}=I+2 i y(B-z)^{-1} .
\end{aligned}
$$

Then define the unitary operator $U(x, y)=T_{A} T_{B}^{*}$ and compute

$$
U-I=T_{A} T_{B}^{*}-T_{B} T_{B}^{*}=\left(T_{A}-T_{B}\right) T_{B}^{*}=i 2 y\left[(A-z)^{-1}-(B-z)^{-1}\right] T_{B}^{*}
$$

which gives

$$
U(x, y)=I+i 2 y(A-z)^{-1}(B-A)(B-\bar{z})^{-1} .
$$

Suppose now that $B-A$ is a nonnegative one-dimensional operator:

$$
B-A=\alpha\langle\cdot, w\rangle w, \quad \text { where } \alpha>0,\|w\|=1 .
$$

Then

$$
U=I+i 2 y \alpha\left\langle\cdot,(B-z)^{-1} w\right\rangle(A-z)^{-1} w .
$$

Taking $v=(A-z)^{-1} w$, we find

$$
U v=\left(1+i 2 y \alpha\left\langle(A-z)^{-1} w,(B-z)^{-1} w\right\rangle\right) v
$$


which shows that $U$ has an eigenvalue $1+\alpha(x, y)$ with

$$
\alpha(x, y)=2 i y \alpha\left\langle(A-z)^{-1} w,(B-z)^{-1} w\right\rangle
$$

The unitary operator $U$ has exactly two eigenvalues, 1 and $1+\alpha(x, y)$, as $B-A$ has exactly two eigenvalues, 0 and $\alpha$. Because of this, $\alpha(x, y) \neq 0$ for all $x \in \mathbb{R}, y>0$. If $\alpha(x, y)=0$ for some $x, y$, then $U(x, y)$ has only one eigenvalue 1 and $U(x, y)=I$ which is impossible, since $A \neq B$. Therefore we can write

$$
1+\alpha(x, y)=e^{i 2 \pi \theta(x, y)},
$$

where $\theta(x, y)$ is a continuous function on the upper half plane with $0<\theta<1$. The unitary operator $U$ itself has the representation $U=e^{i 2 \pi H}$, with $H$ a selfadjoint trace class operator, having two eigenvalues, 0 and $\theta$. Using the logarithm with argument in $(0,2 \pi)$, we can write

$$
i 2 \pi H=\log U, \quad i 2 \pi \theta=\operatorname{tr} \log U=\log (1+\alpha(x, y)) .
$$

Set

$$
X=2 \arctan \frac{A-x}{y}, \quad Y=2 \arctan \frac{B-x}{y} .
$$

Spectral theory easily gives

$$
T_{A}=e^{-i X}, \quad T_{B}=e^{-i Y} .
$$

For large $y>0$ the operators $X, Y$ have small norms and by Lemma 1.1,

$$
i 2 \pi \theta=\operatorname{tr} \log \left(e^{-i X} e^{i Y}\right)=i \operatorname{tr}(Y-X)=i 2 \pi h,
$$

that is, $\theta(x, y)=h(x, y)$. Since $\theta(x, y)$ is harmonic for large $y$, it is harmonic for all $y>0$ because it has the same structure for all $y>0$,

$$
\theta(x, y)=\frac{1}{2 \pi i} \log \left(1+2 i y \alpha\left\langle(A-z)^{-1} w,(B-z)^{-1} w\right\rangle\right) .
$$

We conclude that $\theta(x, y)=h(x, y)$ on the whole upper half plane because both functions are defined and harmonic there. Therefore $0<h<1$. By Fatou's theorem it has boundary values $\xi(x)=\lim _{y \rightarrow 0+} h(x, y)$ a.e. with $0 \leq \xi \leq 1$ and

$$
h(x, y)=\frac{1}{\pi} \int_{\mathbb{R}} \frac{y}{y^{2}+(x-t)^{2}} \xi(t) d t .
$$

From (3.8),

$$
\lim _{y \rightarrow \infty} \pi y h(x, y)=\int_{\mathbb{R}} \xi(t) d t=\|\xi\|_{1} \leq\|B-A\|_{1} .
$$

When $\alpha<0$ we can change the places of $A, B$ and define $\xi(t ; B, A)=-\xi(t ; A, B) \geq 0$, so that in this case $\xi(t ; A, B) \leq 0$. For completeness, if $\alpha=0$ we set $\xi(t ; A, B)=0$. 
In order to define $\xi$ for an arbitrary trace class perturbation

$$
B-A=\sum_{k=1}^{\infty} \alpha_{k}\left\langle\cdot, w_{k}\right\rangle w_{k}, \quad\|B-A\|_{1}=\sum_{k=1}^{\infty}\left|\alpha_{k}\right|<\infty,
$$

we proceed by the staircase method. Let

$$
B_{n}=A+\sum_{k=1}^{n} \alpha_{k}\left\langle\cdot, w_{k}\right\rangle w_{k}, \quad \lim _{n \rightarrow \infty}\left\|B-B_{n}\right\|_{1}=0 .
$$

Suppose that we have defined $\xi\left(t ; A, B_{n}\right)$ for some $n$ with

$$
\begin{aligned}
\left\|\xi\left(t ; A, B_{n}\right)\right\|_{1} & \leq\left\|B_{n}-A\right\|_{1}, \\
\operatorname{tr}\left[\arctan \frac{B_{n}-x}{y}-\arctan \frac{A-x}{y}\right] & =\int_{\mathbb{R}} \frac{y}{y^{2}+(x-t)^{2}} \xi\left(t ; A, B_{n}\right) d t .
\end{aligned}
$$

Then we set

$$
\begin{gathered}
\xi\left(t ; A, B_{n+1}\right)=\xi\left(t ; A, B_{n}\right)+\xi\left(t ; B_{n}, B_{n+1}\right), \\
\left\|\xi\left(t ; A, B_{n+1}\right)\right\|_{1} \leq\left\|\xi\left(t ; A, B_{n}\right)\right\|_{1}+\left\|\xi\left(t ; B_{n}, B_{n+1}\right)\right\|_{1} \leq \sum_{k=1}^{n+1}\left|\alpha_{k}\right|=\left\|B_{k+1}-A\right\|_{1}
\end{gathered}
$$

and (3.27) holds for $n+1$ because we can add and subtract $\arctan \left[\left(B_{n+1}-x\right) / y\right]$ in the left-hand side. By induction, the functions $\xi\left(t ; A, B_{n}\right)$ are defined for all $n$ and it is trivial to see that they form a Cauchy sequence in $L^{1}(\mathbb{R})$. The limit

$$
\xi(t)=\xi(t ; A, B)=\lim \xi\left(t ; A, B_{n}\right)
$$

exists with $\|\xi(t)\|_{1} \leq\|B-A\|_{1}$.

Proof of (c). When $B-A \geq 0$, then all $\alpha_{k} \geq 0$ and in view of (3.28) we find by induction $\forall n: \xi\left(t ; A, B_{n}\right) \geq 0$. Therefore $\xi \geq 0$.

Proof of (a). By (3.4), the following estimate is true

$$
\left\|\arctan \frac{B-x}{y}-\arctan \frac{B_{n}-x}{y}\right\|_{1} \leq \frac{1}{y}\left\|B-B_{n}\right\|_{1} \text {. }
$$

Passing to limits in (3.27), we find

$$
\operatorname{tr}\left[\arctan \frac{B-x}{y}-\arctan \frac{A-x}{y}\right]=\int_{\mathbb{R}} \frac{y}{y^{2}+(x-t)^{2}} \xi(t) d t .
$$

This relation, true for all $x \in \mathbb{R}, y>0$ implies

$$
\operatorname{tr}[f(B)-f(A)]=\int_{\mathbb{R}} f^{\prime}(t) \xi(t) d t
$$

for all functions $f \in K$. Indeed, given $f(t)=\int_{\mathbb{R}}\left(e^{i s t}-1\right) / s d \mu(s)$ define

$$
f(t ; y)=\int_{\mathbb{R}} \frac{e^{i s t}-1}{s} e^{-y|s|} d \mu(s), \quad y>0 .
$$


We have

$$
\begin{aligned}
\frac{d}{d t} f(t ; y) & =f^{\prime}(t ; y)=i \int_{\mathbb{R}} e^{i s t} e^{-y|s|} d \mu(s) \\
& =i \int_{\mathbb{R}}\left[\frac{1}{\pi} \int_{\mathbb{R}} \frac{y}{y^{2}+(x-t)^{2}} e^{i s x} d x\right] d \mu(s) \\
& =\frac{1}{\pi} \int_{\mathbb{R}} \frac{y}{y^{2}+(x-t)^{2}}\left[i \int_{\mathbb{R}} e^{i s x} d \mu(s)\right] d x \\
& =\frac{1}{\pi} \int_{\mathbb{R}} \frac{y}{y^{2}+(x-t)^{2}} f^{\prime}(x) d x .
\end{aligned}
$$

Integrating for $t$ and adjusting the constant of integration so that $f(0, y)=0$, we find

$$
f(t ; y)=\frac{1}{\pi} \int_{\mathbb{R}}\left[\arctan \frac{t-x}{y}+\arctan \frac{x}{y}\right] f^{\prime}(x) d x
$$

therefore,

$$
\begin{aligned}
\operatorname{tr}[f(B ; y)-f(A ; y)] & =\frac{1}{\pi} \int_{\mathbb{R}} \operatorname{tr}\left[\arctan \frac{B-x}{y}-\arctan \frac{A-x}{y}\right] f^{\prime}(x) d x \\
& =\int_{\mathbb{R}}\left[\frac{1}{\pi} \int_{\mathbb{R}} f^{\prime}(x) \frac{y}{y^{2}+(x-t)^{2}} d x\right] \xi(t) d t .
\end{aligned}
$$

Taking limits for $y \rightarrow 0+$ we come to (3.33).

The limit

$$
\lim _{y \rightarrow 0+} \operatorname{tr}[f(B ; y)-f(A ; y)]=\operatorname{tr}[f(B)-f(A)]
$$

becomes obvious when we compare

$$
\operatorname{tr}[f(B ; y)-f(A ; y)]=\int_{\mathbb{R}} \operatorname{tr}\left[\frac{e^{i s B}-e^{i s A}}{s}\right] e^{-y|s|} d \mu(s)
$$

with

$$
\operatorname{tr}[f(B)-f(A)]=\int_{\mathbb{R}} \operatorname{tr}\left[\frac{e^{i s B}-e^{i s A}}{s}\right] d \mu(s) .
$$

The function $f(t)=t$ belongs to $K$ with $d \mu(s)=-i \delta(s) d s$. This gives

$$
\operatorname{tr}(B-A)=\int_{\mathbb{R}} \xi(t) d t .
$$

Proof OF (d). Suppose $\sigma(A) \bigcup \sigma(B) \subseteq[a, b]$ and $x<a$. The relation

$$
\arctan t=\frac{\pi}{2}-\arctan \frac{1}{t} \quad(t>0)
$$

gives (by using the spectral theorem with integration over $(-\infty, x]$ and $[x,+\infty)$ )

$$
\arctan \frac{B-x}{y}-\arctan \frac{A-x}{y}=\arctan \left[y(A-x)^{-1}\right]-\arctan \left[y(B-x)^{-1}\right] .
$$

Therefore,

$$
\xi(x)=\lim _{y \rightarrow 0+} \frac{1}{\pi} \operatorname{tr}\left[\arctan \left(y(A-x)^{-1}\right)-\arctan \left(y(B-x)^{-1}\right)\right]=0 .
$$


The case $x>b$ is treated similarly, using the relation

$$
\arctan t=-\frac{\pi}{2}-\arctan \frac{1}{t} \quad(t<0) .
$$

Moreover, if the spectra of the operators are separated, it easily follows that on intervals between them the SSF is a constant. The proof is completed.

REMARK 3.1. The above proof allows a natural extension of KreĬn's formula. Let $A$ and $B$ be the generators of one-parameter $C_{0}$-groups of operators: $e^{i t A}, e^{i t B}, t \in \mathbb{R}$, of at most polynomial growth

$$
\left\|e^{i t A}\right\|,\left\|e^{i t B}\right\| \leq M(1+|t|)^{\alpha}, \quad \alpha \geq 0
$$

(when $M=1, \alpha=0$, the operators are selfadjoint). The harmonic function (3.7) is well defined and its boundary value (2.6) is a certain distribution for which (2.4) holds.

4. Admissible functions. Let $A, B$ be two selfadjoint operators with $B-A \in S_{1}$. One differentiable function $f(x)$ defined on some interval containing $\sigma(A) \cup \sigma(B)$ is called admissible, if $f(B)-f(A) \in S_{1}$ and the trace formula (2.4) holds. We proved that the functions in the set $K$ are admissible. Obviously, if $f$ is admissible, then $f+c$ is also admissible for any constant $c$. Any linear combination of admissible functions is admissible. One could expect that every function with $f^{\prime} \in L_{\infty}(\mathbb{R})$ is admissible. However, Farforovskaya produced an example of a function $f$ with bounded continuous derivative and a pair of selfadjoint operators $A, B$ such that $B-A \in S_{1}$ but $f(B)-f(A) \notin S_{1}$ (see [11] and the note at the end of it). The characterization of all admissible functions is an open problem. Birman and Solomyak [5], using the methods of double operator integrals, described a large class of admissible functions, including those with $f^{\prime} \in L_{p}(\mathbb{R}) \bigcap \operatorname{Lip} \epsilon$, where $1 \leq p<\infty, \epsilon>0$. Their investigations were continued by Peller [24, 25], who showed that every function in the Besov class $B_{\infty, 1}^{1}$ is admissible. Using only simple means, we want to give here some examples of admissible functions, besides those in $K$.

Proposition 4.1 [20]. Suppose $v(t)$ is a finite measure on a set $M \subseteq \mathbb{R}$ such that

$$
\int_{M}|t| d|v|(t)<\infty
$$

Then all functions of the form $f(x)=\int_{M} e^{i t x} d v(t)$ are admissible.

Proof. Writing the trace formula for the admissible function

$$
f_{t}(x)=\frac{e^{i t x}-1}{t}
$$

we get

$$
\operatorname{tr}\left(e^{i t B}-e^{i t A}\right)=\int_{\mathbb{R}} i t e^{i t x} \xi(x) d x
$$

which shows that the function $g_{t}(x)=e^{i t x}$ is admissible. In view of (3.2), we can multiply both sides in (4.3) by $d v$ and integrate over $M$. 
COROLlary 4.2. When $\operatorname{Im}(z) \neq 0$ the function $f_{z}(x)=1 /(x+z)$ and all its derivatives are admissible.

Proof. Let $z=s+i t$. For $t>0$, we write

$$
\frac{1}{x+s+i t}=\frac{-i}{-i x-i s+t}=-i \int_{0}^{\infty} e^{i \lambda x} e^{i \lambda s} e^{-\lambda t} d \lambda
$$

and for $t<0$,

$$
\frac{1}{x+s+i t}=\frac{i}{i x+i s-t}=i \int_{0}^{\infty} e^{-i \lambda x} e^{-i s \lambda} e^{\lambda t} d \lambda .
$$

The result follows immediately from here. We deduce that the function

$$
f_{t}(x)=\frac{x}{x^{2}+t^{2}}=\frac{1}{2}\left[\frac{1}{x+i t}+\frac{1}{x-i t}\right]
$$

is also admissible.

Now we turn to the case of nonnegative operators $A, B$. In view of property (d) we need to consider only functions on $[0, \infty)$.

Proposition 4.3. Let $0 \leq A, B$ and $v(t)$ be a finite measure on $[0, \infty)$ with

$$
\int_{0}^{\infty}|t| d|v|(t)<\infty
$$

Then all functions of the form

$$
f(x)=\int_{0}^{\infty} e^{-t x} d v(t), \quad x \geq 0
$$

are admissible.

Proof. When $t, x>0$ the function $f_{t}(x)=e^{-t x}$ is admissible, as seen from

$$
e^{-t x}=1+\frac{t^{2}}{\pi} \int_{\mathbb{R}} \frac{e^{-i s x}-1}{i s} \frac{d s}{t^{2}+s^{2}}
$$

(to check this, differentiate both sides for $x$ ). Then one proceeds as in Proposition 4.1, integrating

$$
\operatorname{tr}\left(e^{-t B}-e^{-t A}\right)=-\int_{0}^{\infty} t e^{-t x} \xi(x) d x
$$

Proposition 4.4. Suppose $0<\epsilon I \leq A, B$ and $f(x)$ is a function on $(0, \infty)$ that admits a bounded holomorphic extension $f(z)$ on the right half plane $\operatorname{Re}(z)>0$. Then $f$ is admissible for $A, B$.

Proof. One has the Poisson representation

$$
f(x)=\frac{1}{\pi} \int_{\mathbb{R}} f(i t) \frac{x}{x^{2}+t^{2}} d t,
$$

where $f(i t)$ is the boundary value of $f(z)$ defined a.e. The spectral theorem gives

$$
f(A)=\frac{1}{\pi} \int_{\mathbb{R}} f(i t) \frac{A}{A^{2}+t^{2}} d t .
$$


In the same way we represent $f(B)$. Since the function $x /\left(x^{2}+t^{2}\right)$ is admissible, one can write

$$
\operatorname{tr}\left[\frac{B}{B^{2}+t^{2}}-\frac{A}{A^{2}+t^{2}}\right]=\int_{\mathbb{R}} \frac{t^{2}-x^{2}}{\left(x^{2}+t^{2}\right)^{2}} \xi(x) d x .
$$

Multiplying both sides by $f(i t)$ and integrating over $\mathbb{R}$ one comes to (2.4). To see that the integral on the left side converges, one needs to check that $f(B)-f(A) \in S_{1}$. Indeed,

$$
\begin{aligned}
\left\|\frac{1}{B+i t}-\frac{1}{A+i t}\right\|_{1} & =\left\|\frac{1}{B+i t}(B-A) \frac{1}{A+i t}\right\|_{1} \\
& \leq\left\|\frac{1}{B+i t}\right\|\|\| \frac{1}{A+i t}\|\| B-A\left\|_{1} \leq \frac{1}{(\epsilon+|t|)^{2}}\right\| B-A \|_{1} .
\end{aligned}
$$

Using the decomposition (4.6), one estimates

$$
\left\|\frac{B}{B^{2}+t^{2}}-\frac{A}{A^{2}+t^{2}}\right\|_{1} \leq \frac{1}{(\epsilon+|t|)^{2}}\|B-A\|_{1}
$$

and therefore,

$$
\|f(B)-f(A)\|_{1} \leq \frac{2}{\pi \epsilon} \sup _{\operatorname{Re}(z)>0}|f(z)|\|B-A\|_{1} .
$$

EXAMPLE 4.5. Taking $f(x)=x^{i s}, s \in \mathbb{R}$, one finds

$$
\operatorname{tr}\left(B^{i s}-A^{i s}\right)=i s \int_{\epsilon}^{\infty} x^{i s-1} \xi(x) d x .
$$

REMARK 4.6. In Proposition 4.4, one may assume only that $f(x)$ admits a bounded holomorphic extension on some sector $|\operatorname{Arg}(z)|<\pi / 2$. The estimate (4.16) can be improved by using an appropriate integral representation of such function [9].

5. $\Phi$-compatible operators. It may happen that the difference $B-A$ is not trace class, but for some common regular point $z$,

$$
(B-z)^{-1}-(A-z)^{-1} \in S_{1} .
$$

Such operators are called resolvent compatible. If (5.1) is true for some $z \in \rho(A) \cap \rho(B)$, then it is true for all $\lambda \in \rho(A) \cap \rho(B)$, as follows from the identity

$$
(B-\lambda)^{-1}-(A-\lambda)^{-1}=(B-z)(B-\lambda)^{-1}\left[(B-z)^{-1}-(A-z)^{-1}\right](A-z)(A-\lambda)^{-1} .
$$

An important case is when the operators are bounded from below. We may assume that $0 \leq A, B$. Then (5.1) is equivalent to

$$
(B+I)^{-1}-(A+I)^{-1} \in S_{1}
$$

and we can apply the trace formula to the operators $(B+I)^{-1}$ and $(A+I)^{-1}$. After that the substitution $t \rightarrow t^{-1}-1$ brings to a trace formula for $A, B$.

More generally, we have the following. 
DEFINITION 5.1. Let $\Phi$ be a real-valued continuous function on some finite or infinite interval $[a, b]$ with $\Phi^{\prime}$ existing and nonzero on $(a, b)$. Two selfadjoint operators $A, B$ with spectra in $[a, b]$ are called $\Phi$-compatible, if

$$
\Phi(B)-\Phi(A) \in S_{1} .
$$

Kreǐn's trace formula extends to such operators by a simple substitution.

Corollary 5.2. Suppose $\Phi$ is as above and $A, B$ are $\Phi$-compatible. There exists a spectral shift function $\xi$ defined a.e. on $[a, b]$ for which

$$
\operatorname{tr}[f(B)-f(A)]=\int_{a}^{b} f^{\prime}(t) \xi(t) d t
$$

for any differentiable function $f$ on $[a, b]$ such that $f\left(\Phi^{-1}(x)\right)$ is admissible for the interval $[\Phi(a), \Phi(b)]$. Property (d) stays the same, while (b) turns into

$$
\int_{a}^{b}\left|\xi(t) \Phi^{\prime}(t)\right| d t \leq\|\Phi(B)-\Phi(A)\|_{1} .
$$

Proof. Write the trace formula (2.6) for the pair $\Phi(A), \Phi(B)$ and define

$$
\xi(t)=\xi(\Phi(t) ; \Phi(A) ; \Phi(B)) .
$$

Then the substitution $x=\Phi(t)$ brings to (5.5).

6. Proof of Lemma 1.1 (the Baker-Campbell-Hausdorff formula). It is known that if $X, Y \in B(H)$, then an infinite series $Z=Z(X, Y)$ exists such that

$$
e^{Z}=e^{X} e^{Y}
$$

For instance (see [8, Chapters 1 and 2], [23, 30]),

$$
Z=X+Y+\sum_{n \geq 2} \frac{1}{n} \sum_{|w|=n} g_{w}[w],
$$

where $g_{w}$ are certain coefficients and $w=w_{1} w_{2} \cdots w_{n}$ is a "word" with length $|w|=n, n=2,3, \ldots$, such that each $w_{K}$ equals $X$ or $Y$. Also, $[w]$ is the iterated commutator

$$
[w]=\left[\left[\cdots\left[\left[w_{1}, w_{2}\right], w_{3}\right] \cdots\right], w_{n}\right] .
$$

This series was studied by Thompson [30], who proved its convergence when $X, Y$ have small norms. Details and precise statements can be found in his paper (see also [23]). A modification of Thompson's proof yields the following.

Proof of Lemma 1.1. If $X+Y \in S_{1}$, then $[X, Y] \in S_{1}$ too and $\operatorname{tr}[X, Y]=0$, as $[X, Y]=[X+Y, Y]$. The trace of all higher commutators is also zero. Now recall that $\|A B\|_{1} \leq\|A\|\|B\|_{1}$ for any two operators $A \in B(H), B \in S_{1}$. We set $\delta=\max \{\|X\|,\|Y\|\}$ and estimate

$$
\begin{gathered}
\|[X, Y]\|_{1}=\|[X+Y, Y]\|_{1} \leq 2\|Y\|\|X+Y\|_{1} \leq 2 \delta\|X+Y\|_{1}, \\
\|[[X, Y], X]\|_{1} \leq 2\|X\|\|[X, Y]\|_{1} \leq 2^{2} \delta^{2}\|X+Y\|_{1}
\end{gathered}
$$


and so forth. By induction, for every $n \geq 2$,

$$
\|[w]\|_{1} \leq 2^{n-1} \delta^{n-1}\|X+Y\|_{1}
$$

whenever $|w|=n$. Combining this with Thompson's estimates [30, pages 5 and 6], we find

$$
\left\|\sum_{|w|=n} g_{w}[w]\right\|_{1} \leq 2^{n} \delta^{n-1}\|X+Y\|_{1} .
$$

The series in (6.2) is majorized in the norm of $S_{1}$ by

$$
\|X+Y\|_{1} \sum_{n \geq 2} \frac{2^{n} \delta^{n-1}}{n}
$$

which is convergent, since $\delta<1 / 2$. Therefore, the expansion (6.2) converges in $S_{1}$ and the proof is completed.

\section{REFERENCES}

[1] V. M. Adamjan and H. Neidhardt, On the summability of the spectral shift function for pair of contractions and dissipative operators, J. Operator Theory 24 (1990), no. 1, 187-205. MR 92d:47016. Zbl 795.47023.

[2] H. Baumgärtel and M. Wollenberg, Mathematical Scattering Theory, Mathematische Lehrbucher und Monographien, II. Abteilung: Mathematische Monographien [Mathematical Textbooks and Monographs, Part II: Mathematical Monographs], vol. 59, Akademie-Verlag, Berlin, 1983. MR 86c:47006a. Zbl 536.47007.

[3] M. Š. Birman and M. G. Kreĭn, On the theory of wave operators and scattering operators, Soviet Math. Dokl. 3 (1962), 740-744 (Russian), [translation from Dokl. Akad. Nauk SSSR 144 (1962), 475-478. MR 25\#2447]. Zbl 196.45004.

[4] M. Š. Birman and A. B. Pushnitski, Spectral shift function, amazing and multifaceted, Integral Equations Operator Theory 30 (1998), no. 2, 191-199, dedicated to the memory of Mark Grigorievich Kreǐn (1907-1989). MR 98m:47012. Zbl 907.47014.

[5] M. Š. Birman and M. Z. Solomyak, Remarks on the spectral shift function. Boundary value problems of mathematical physics and related questions in the theory of functions, 6, J. Soviet Math. 3 (1975), 408-419 (Russian), [translation from Zap. Naučn. Sem. Leningrad. Otdel. Mat. Inst. Steklov. (LOMI) 27 (1972), 33-46. MR 47\#4031]. Zbl 336.47017.

[6] M. Š. Birman and D. R. Yafaev, The spectral shift function. The papers of M. G. Krĕn and their further development, St. Petersbg. Math. J. 4 (1993), no. 5, 833-870 (Russian), [translation from Algebra i Analiz 4 (1992), no. 5, 1-44. MR 94g:47002]. Zbl 791.47013.

[7] D. Bollé, F. Gesztesy, H. Grosse, W. Schweiger, and B. Simon, Witten index, axial anomaly, and Krĕn's spectral shift function in supersymmetric quantum mechanics, J. Math. Phys. 28 (1987), no. 7, 1512-1525. MR 88j:81022. Zbl 643.47005.

[8] N. Bourbaki, Elements of Mathematics. Lie Groups and Lie Algebras. Part I: Chapters 1-3, Adiwes International Series in Mathematics, Addison-Wesley Publishing Company, Reading, Mass., 1975, [translated from the original French edition, Actualites Scientifiques et Industrielles, Hermann, Paris, 1975. MR 56\#12077]. Zbl 319.17002.

[9] K. N. Boyadzhiev, Norm inequalities in perturbation theory, in preparation.

[10] _ Mean value theorems for traces, Math. Japon. 38 (1993), no. 2, 217-224. MR 94d:46067. Zbl 812.46051.

[11] J. B. Farforovskaja, An example of a Lipschitzian function of selfadjoint operators that yields a nonnuclear increase under a nuclear perturbation, J. Soviet Math. 4 (1975), 
426-433 (Russian), [translation from Zap. Naučn. Sem. Leningrad. Otdel. Mat. Inst. Steklov. (LOMI) 30 (1972), 146-153. MR 49\#1175. Zbl 333.47009].

[12] R. Geisler, V. Kostrykin, and R. Schrader, Concavity properties of Krěn's spectral shift function, Rev. Math. Phys. 7 (1995), no. 2, 161-181. MR 96a:47024. Zbl 836.47049.

[13] F. Gesztesy, K. A. Makarov, and E. Tsekanovskii, An addendum to Krěn's formula, J. Math. Anal. Appl. 222 (1998), no. 2, 594-606. MR 99g:47047. Zbl 922.47006.

[14] F. Gesztesy and B. Simon, Topological invariance of the Witten index, J. Funct. Anal. 79 (1988), no. 1, 91-102. MR 90a:47032. Zbl 649.47012.

[15] _ The $\xi$ function, Acta Math. 176 (1996), no. 1, 49-71. MR 97e:47078. Zbl 885.34070.

[16] P. Jonas, On the trace formula of perturbation theory. I, Math. Nachr. 137 (1988), 257-281. MR 89k:47020. Zbl 683.47008.

[17] P. Koosis, Introduction to $H_{p}$ Spaces, 2nd ed., Cambridge Tracts in Mathematics, vol. 115, Cambridge University Press, Cambridge, New York, 1998, with two appendices by V. P. Havin [Viktor Petrovich Khavin]. MR 2000b:30052. Zbl 990.03992.

[18] V. Kostrykin and R. Schrader, Cluster properties of one-particle Schrödinger operators, Rev. Math. Phys. 6 (1994), no. 5, 833-853. MR 95k:81145. Zbl 813.47011.

[19] M. G. Krein, On the trace formula in perturbation theory, Mat. Sbornik (N.S.) 33(75) (1953), 597-626 (Russian). MR 15,720b. Zbl 052.12303.

[20] - On perturbation determinants and a trace formula for unitary and self-adjoint operators, Soviet Math. Dokl. 3 (1962), 707-710, [translation from Dokl. Akad. Nauk SSSR 144 (1962), 268-271. MR 25\#2446]. Zbl 191.15201.

[21] _ Topics in Differential and Integral Equations and Operator Theory, Operator Theory: Advances and Applications, vol. 7, Birkhäuser Verlag, Basel, Boston, Mass., 1983, Edited by I. Gohberg. Translated from the Russian by A. Iacob. MR 86m:00014. Zbl 512.45001.

[22] I. M. Lifšic, On a problem of the theory of perturbations connected with quantum statistics, Uspehi Matem. Nauk (N.S.) 7 (1952), no. 1(47), 171-180 (Russian). MR 14,185b. Zbl 046.21203.

[23] M. Newman, W. So, and R. C. Thompson, Convergence domains for the Campbell-BakerHausdorff formula, Linear and Multilinear Algebra 24 (1989), no. 4, 301-310. MR 91e:17028. Zbl 713.22007.

[24] V. V. Peller, Hankel operators in the theory of perturbations of unitary and selfadjoint operators, Funct. Anal. Appl. 19 (1985), 111-123, [translation from Funktsional. Anal. i Prilozhen 19 (1985), no. 2, 37-51. MR 87e:47029]. Zbl 587.47016.

[25] - Hankel operators in the perturbation theory of unbounded selfadjoint operators, Analysis and Partial Differential Equations, pp. 529-544, Lecture Notes in Pure and Appl. Math., vol. 122, Dekker, New York, 1990. MR 92d:47037. Zbl 716.47015.

[26] A. V. Rybkin, The spectral shift function, the characteristic function of a contraction and a generalized integral, Russian Acad. Sci. Sb. Math. 83 (1995), no. 1, 237-281 (Russian), [translation from Mat. Sb. 185 (1994), no. 10, 91-144. MR 96k:47002]. Zbl 852.47004.

[27] B. Simon, Spectral analysis of rank one perturbations and applications, Mathematical Quantum Theory. II. Schrödinger Operators (Vancouver, BC, 1993), pp. 109-149 (J. Feldman, R Froese, and L. M. Rosen, eds.), CRM Proc. Lecture Notes, vol. 8, Amer. Math. Soc., Providence, RI, 1995, Seminar: Mathematical Quantum Theory, Canadian Mathematical Society Annual; Vancouver, BC, 1993. MR 97c:47008. Zbl 824.47019.

[28] K. B. Sinha and A. N. Mohapatra, Spectral shift function and trace formula, Proc. Indian Acad. Sci. Math. Sci. 104 (1994), no. 4, 819-853, Spectral and inverse spectral theory (Bangalore, 1993). MR 96j:47011. Zbl 821.47005.

[29] A. V. Sobolev, Efficient bounds for the spectral shift function, Ann. Inst. H. Poincaré Phys. Théor. 58 (1993), no. 1, 55-83. MR 94c:47018. Zbl 813.47006. 
[30] R. C. Thompson, Convergence proof for Goldberg's exponential series, Linear Algebra Appl. 121 (1989), 3-7, Linear algebra and applications (Valencia, 1987). MR 90i:22011. Zbl 678.22003.

[31] D. Voiculescu, On a trace formula of M. G. Kreı̆n, Operators in Indefinite Metric Spaces, Scattering Theory and other Topics (Bucharest, 1985), pp. 329-332, Oper. Theory: Adv. Appl., vol. 24, Birkhäuser, Basel, Boston, Mass., 1987. MR 88k:47015. Zbl 647.47019.

[32] D. R. Yafaev, Mathematical Scattering Theory. General theory. Translated from the Russian by J. R. Schulenberger, Translations of Mathematical Monographs, vol. 105, American Mathematical Society, Providence, RI, 1992. MR 94f:47012. Zbl 761.47001.

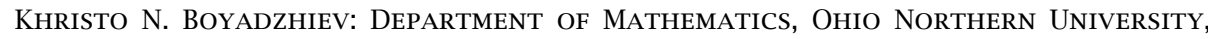
ADA, OHIO 45810, USA

E-mail address: k-boyadzhiev@onu.edu 


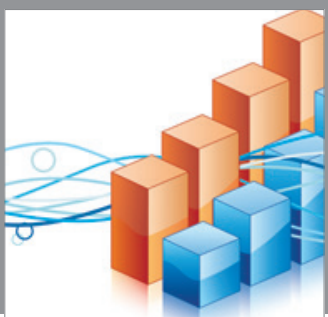

Advances in

Operations Research

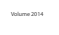

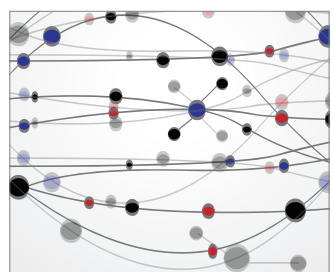

\section{The Scientific} World Journal
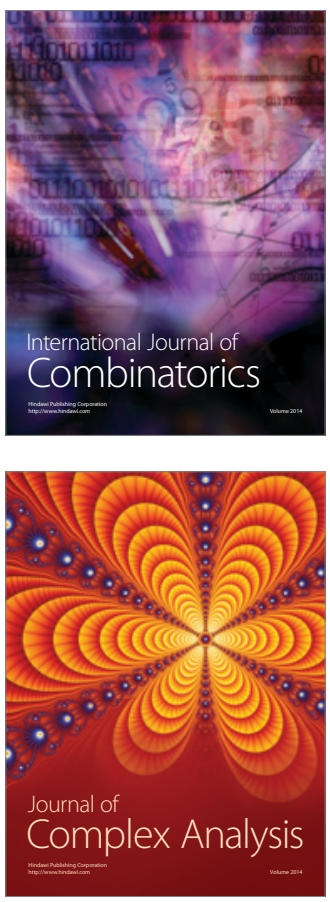

International Journal of

Mathematics and

Mathematical

Sciences
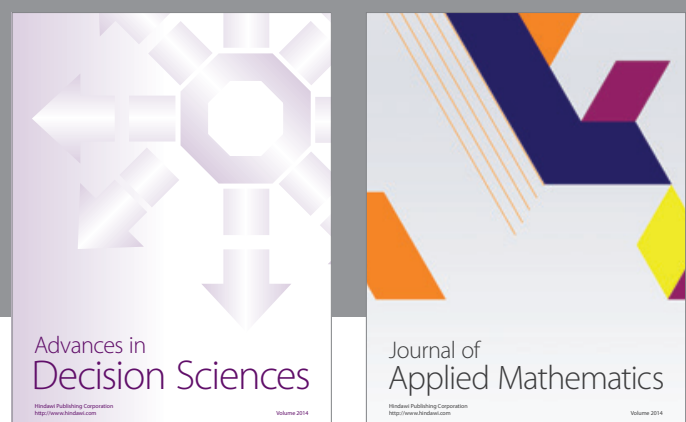

Journal of

Applied Mathematics
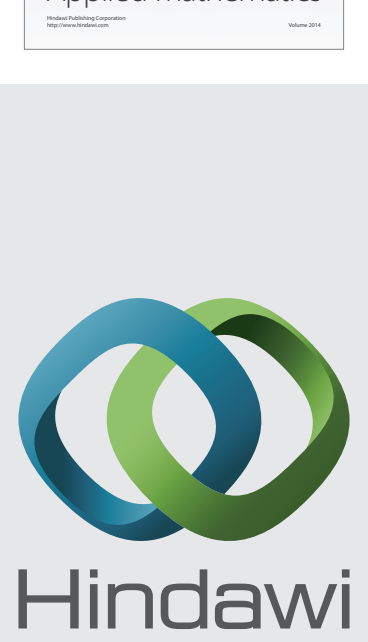

Submit your manuscripts at http://www.hindawi.com
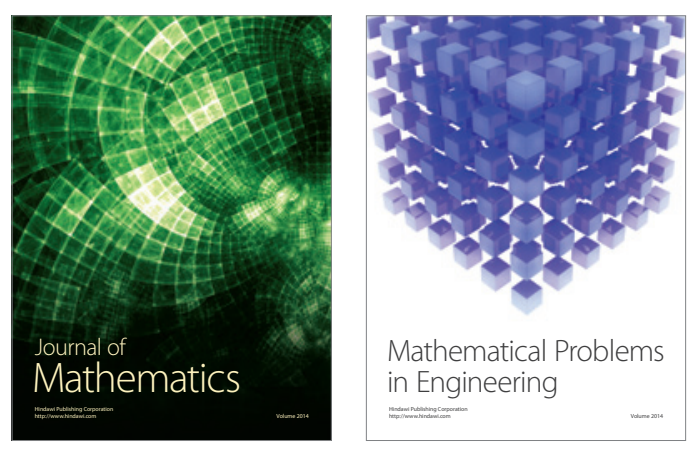

Mathematical Problems in Engineering
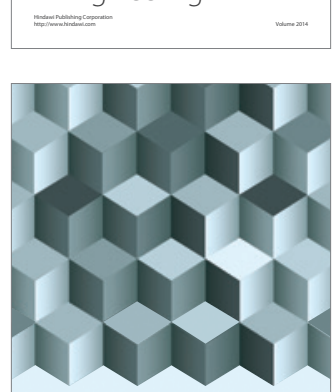

Journal of

Function Spaces
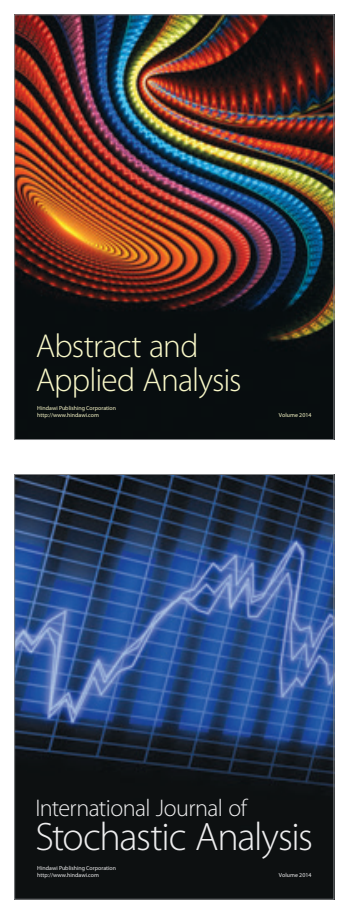

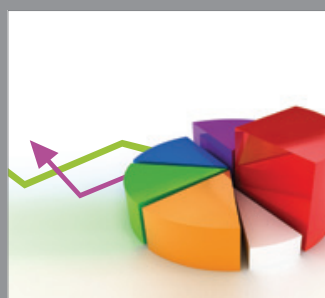

ournal of

Probability and Statistics

Promensencen
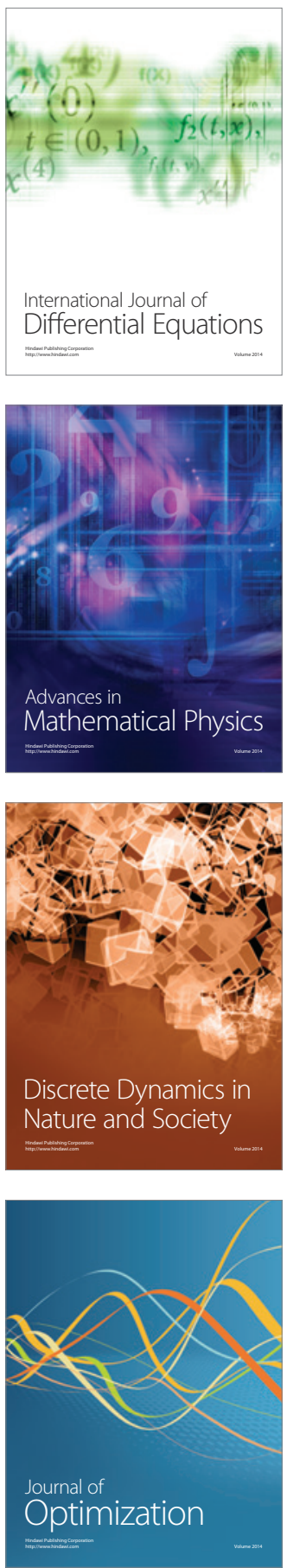